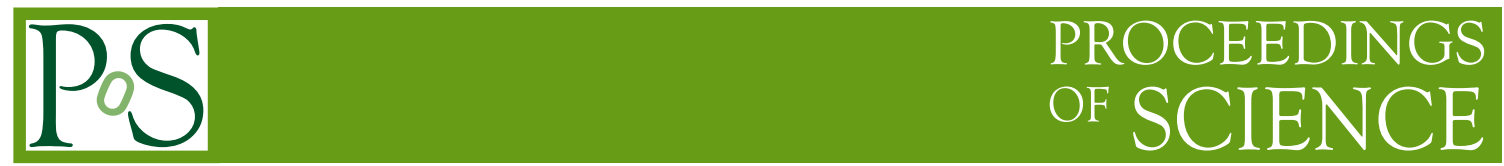

\title{
Physics results from ALICE
}

\author{
Luciano Ramello*t \\ Università del Piemonte Orientale and INFN \\ E-mail: ramello@to.infn.it
}

In this lecture I present the status of experimental search and study of the Quark-Gluon Plasma in $\mathrm{Pb}-\mathrm{Pb}$ collisions at the CERN LHC by the ALICE Collaboration based on the data-taking during years 2010-13 (LHC Run 1), as well as results from pp and p-Pb collisions.

Proceedings of the Corfu Summer Institute 2014 "School and Workshops on Elementary Particle Physics and Gravity",

3-21 September 2014

Corfu, Greece

\footnotetext{
*Speaker.

${ }^{\dagger}$ On behalf of the ALICE Collaboration.
} 


\section{Introduction}

Why are we busy studying heavy-ion collisions at very high energies? Because we want to learn about (i) the non-perturbative aspects of Quantum ChromoDynamics (QCD), namely confinement and breaking/restoration of chiral symmetry, (ii) the QCD phase diagam, and (iii) because we want to recreate in the laboratory the conditions of the phase transition between Quark-Gluon Plasma (QGP) and hadronic matter, which occurred in the early Universe about $10 \mu \mathrm{s}$ after the Big Bang.

The QCD phase diagram as a function of temperature and baryon chemical potential, and particularly the nature of the phase transition (crossover vs. first order) between QGP and hadronic matter, is currently being studied by the Relativistic Heavy Ion Collider (RHIC) experiments and by the Large Hadron Collider (LHC) experiments. The key control parameters in heavy-ion collisions are the collision energy per nucleon pair $\sqrt{s_{\mathrm{NN}}}$, the centrality percentile which is related to the impact parameter $b$, the number of participants $N_{\text {part }}$ and the number of binary collisions $N_{\text {coll }}$ via the Glauber model, and the system size which is in turn dependent on the nuclear species $(\mathrm{Cu}, \mathrm{Au}$, $\mathrm{Pb}, \mathrm{U}$ have been used mostly). The progress achieved over the years in the energy available for particle production form the AGS to LHC is reviewed in [1]. It is interesting to note that the energy doubling time is about 4 years for pp collisions and 1.7 years for heavy-ion collisions.

The first run of the Large Hadron Collider (LHC Run 1) at CERN has been recently completed, including data taking of pp collisions at the center-of-mass energies of 2.76, 7 and $8 \mathrm{TeV}$, two $\mathrm{Pb}-\mathrm{Pb}$ runs at $\sqrt{s_{\mathrm{NN}}}=2.76 \mathrm{TeV}$ (with increasing luminosity) and most recently a $\mathrm{p}-\mathrm{Pb}$ run at $\sqrt{s_{\mathrm{NN}}}=5.02 \mathrm{TeV}$ : see Table 1 . In this contribution, the main ALICE results from the $\mathrm{Pb}-\mathrm{Pb}$ runs of years 2010 and 2011 and from the $\mathrm{p}-\mathrm{Pb}$ one of 2013 are presented.

\begin{tabular}{ccccl} 
System & $\sqrt{s_{\mathrm{NN}}}(\mathrm{TeV})$ & Year & Integrated luminosity & Main goal \\
\hline $\mathrm{Pb}-\mathrm{Pb}$ & 2.76 & 2010 & $10 \mu \mathrm{b}^{-1}$ & First $\mathrm{Pb}-\mathrm{Pb}$ data taking \\
$\mathrm{Pb}-\mathrm{Pb}$ & 2.76 & 2011 & $0.1 \mathrm{nb}^{-1}$ & Study hot \& dense QCD matter \\
$\mathrm{p}-\mathrm{Pb}, \mathrm{Pb}-\mathrm{p}$ & 5.02 & 2013 & $15 \mathrm{nb}^{-1}($ each) & Cold Nuclear Matter effects \\
$\mathrm{pp}$ & 0.9 & $2009-10$ & $0.15 \mathrm{nb}^{-1}$ & Commissioning \\
$\mathrm{pp}$ & 7 & 2010 & $7 \mathrm{nb}^{-1}$ & \\
$\mathrm{pp}$ & 2.76 & 2011 & $1.1 \mathrm{nb}^{-1}$ & Reference for $\mathrm{Pb}-\mathrm{Pb}$ \\
$\mathrm{pp}$ & 7 & 2011 & $4.8 \mathrm{pb}^{-1}$ & \\
$\mathrm{pp}$ & 8 & 2012 & $9.7 \mathrm{nb}^{-1}$ & \\
\hline
\end{tabular}

Table 1: ALICE data samples collected in LHC Run 1.

\section{The ALICE detector}

The ALICE (A Large Ion Collider Experiment) detector has been designed with a capability of handling a charged particle density $\mathrm{d} N_{\mathrm{ch}} / \mathrm{d} \eta$ up to 8000 . ALICE features a central barrel providing tracking and particle identification (PID) of charged particles in a $0.5 \mathrm{~T}$ solenoidal magnetic field, a forward muon arm with a dipole magnet, photon and jet reconstruction with the Photon Spectrometer (PHOS) and the electromagnetic calorimeter (EMCal) and (very) forward detectors, including 
zero degree calorimeters (ZDC), for centrality determination and extended rapidity coverage. The ALICE detector as built for the LHC Run 1 is described in [2].

The central barrel tracking of charged particles in the pseudorapidity range $-0.9<\eta<0.9$ is based mainly on the large Time Projection Chamber (TPC), providing up to 159 samples per track, and on the Internal Tracking System (ITS) made of 6 layers of silicon detectors. The TPCITS transverse momentum resolution is about $1 \%$ at $1 \mathrm{GeV} / c$ and reaches $10 \%$ at $50 \mathrm{GeV} / c$. The material budget has been kept very low so that high precision tracking is possible down to very low transverse momentum $\left(p_{\mathrm{T}}\right)$ : figure 1 presents the track impact parameter $\left(d_{0}\right)$ resolution in the transverse plane $(r \phi)$ as a function of $p_{\mathrm{T}}$ obtained in $\mathrm{pp}, \mathrm{p}-\mathrm{Pb}$ and $\mathrm{Pb}-\mathrm{Pb}$ collisions. The resolution shown includes the contribution from the vertex determination, which is also very precise.

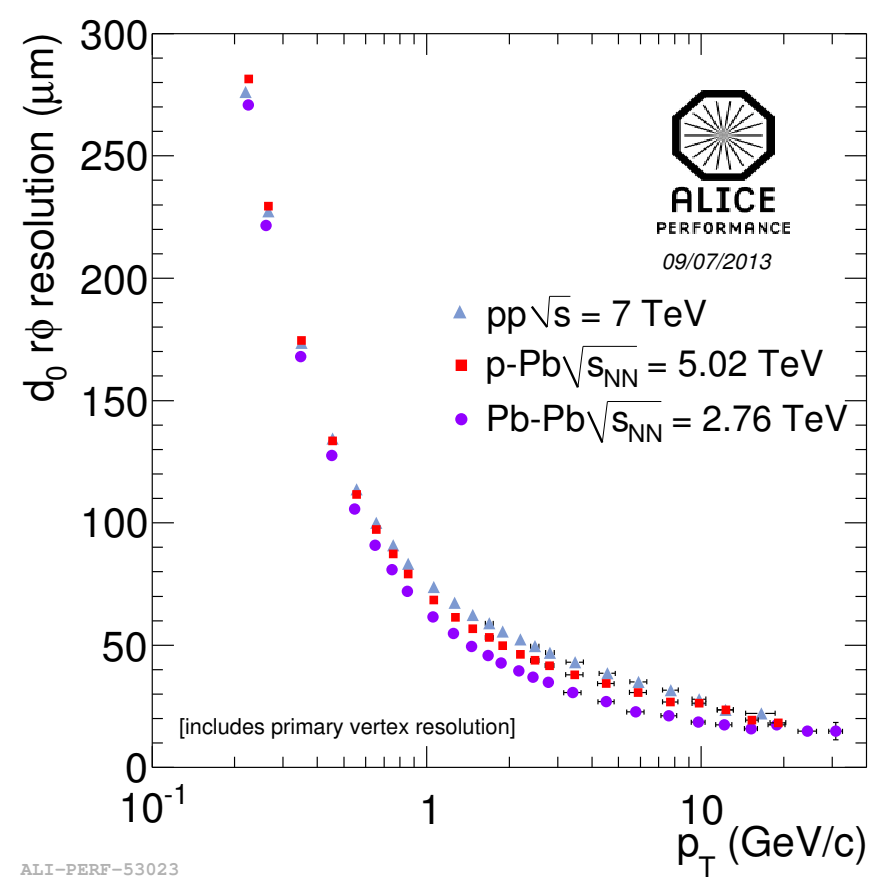

Figure 1: Track impact parameter resolution in the transverse plane vs. $p_{\mathrm{T}}$ for charged particles.

Particle identification in ALICE is performed using essentially all known techniques, only some examples are given in the following. Electron identification is obtained e.g. by a combination of the energy loss $\mathrm{d} E / \mathrm{d} x$ signal in the TPC - compared to the average energy loss expected for electrons - and of the energy/momentum ratio $E / p$ from the energy in the EMCal and the momentum in the TPC, as shown in figure 2.

The particle velocity $\beta$ is obtained from the Time Of Flight (TOF) system using also the track length provided by the TPC and is shown in figure 3, where the bands corresponding to pions, kaons, protons and deuterons are clearly visibile. The momentum range for particle identification is extended using $\mathrm{d} E / \mathrm{d} x$ in the TPC, both towards low momenta (figure 4) and, on a statistical basis, towards high momenta as shown in figure 5. The ITS, having 4 of its layers equipped with analog readout, provides particle identification at even lower momenta (about $100 \mathrm{MeV} / c$ for pions) for those tracks that do not reach the TPC, as shown in figure 6. 


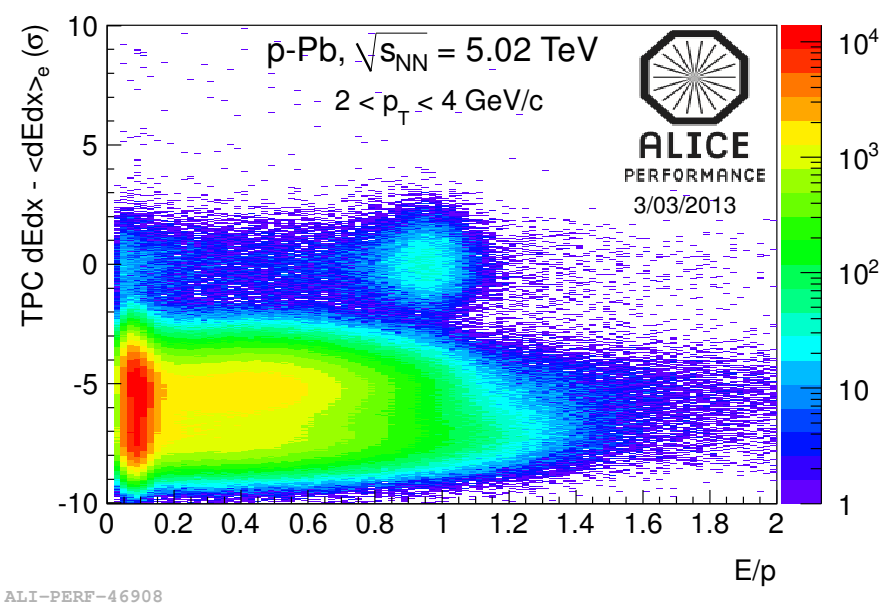

Figure 2: Correlation between $\mathrm{d} E / \mathrm{d} x$ from the TPC (minus the expected electron $\mathrm{d} E / \mathrm{d} x$ ) and $E / p$ from the e.m. calorimeter and the TPC.

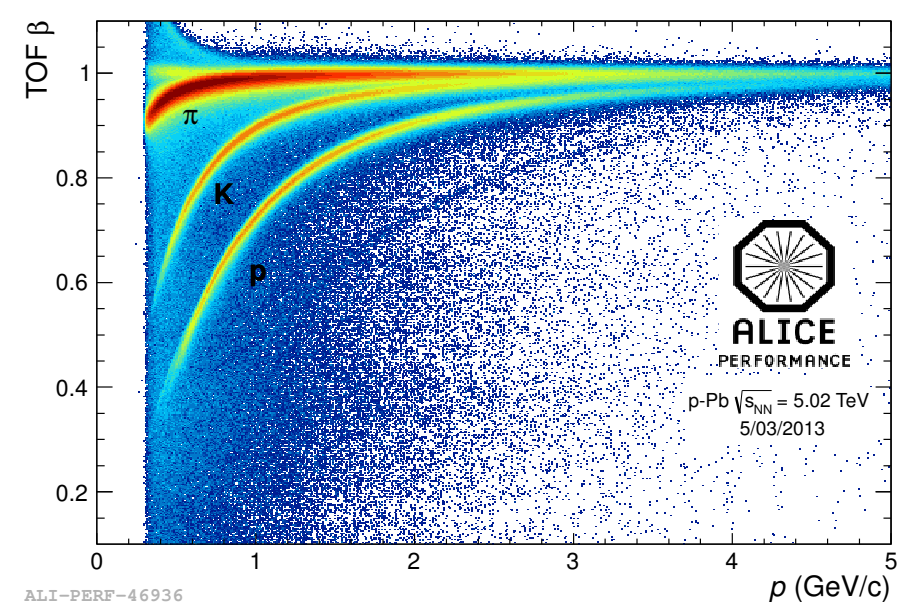

Figure 3: $\beta$ vs. momentum from the Time Of Flight system in $\mathrm{p}-\mathrm{Pb}$ collisions.

Further PID capabilities are provided by the Transition Radiation Detector (TRD) for electron/pion separation and at higher momenta by the HMPID. Another powerful tool is topological particle identification in the TPC which allows us to reconstruct weakly decaying particles such as strange mesons and baryons. Using the TPC $\mathrm{d} E / \mathrm{d} x$ light nuclei and anti-nuclei have also been identified.

The dimuon spectrometer provides muon identification at forward rapidity by filtering out hadrons via a front absorber and a further absorber placed before the muon trigger stations. An example of the invariant mass resolution obtained in $\mathrm{p}-\mathrm{Pb}$ collisions is shown in figure 7 where the spectrum measured in the low mass region is compared with a cocktail including dimuons from resonances, three-body decays of hadrons and semileptonic decays of charm hadrons.

Also the single muon spectrum can be used at high enough $p_{\mathrm{T}}$ to extract the signals from beauty, $\mathrm{W}$ and $\mathrm{Z}$ bosons, as shown in figure 8 . Another example is the extraction of the $J / \psi$ signal from the unlike-sign dimuon invariant mass spectrum, shown in figure 9 , where the $\psi^{\prime}$ peak is also 


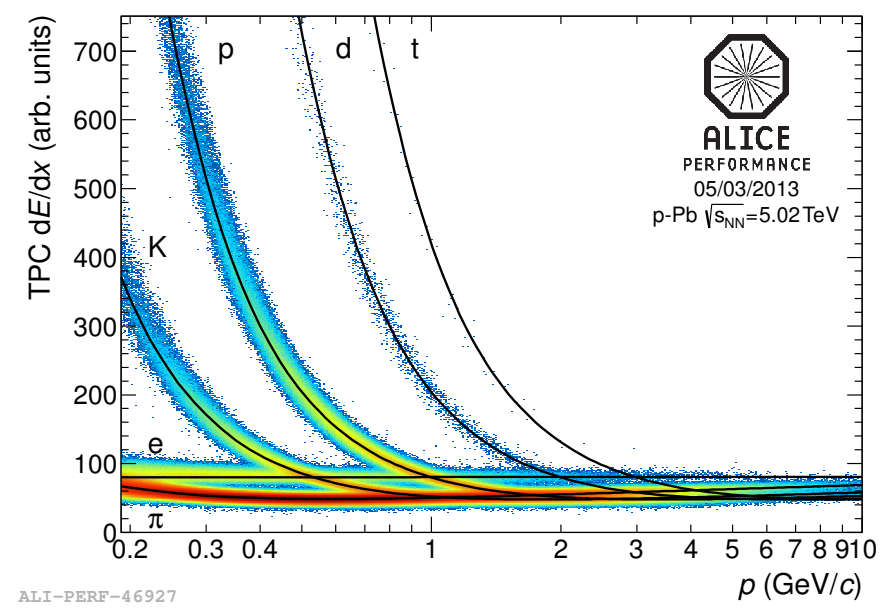

Figure 4: $\mathrm{d} E / \mathrm{d} x$ vs. momentum from the Time Projection Chamber in $\mathrm{p}-\mathrm{Pb}$ collisions.

visibile.

\section{Global properties}

The main control parameter during a run with a given type of collision (e.g. $\mathrm{Pb}-\mathrm{Pb}$ or $\mathrm{p}-\mathrm{Pb}$ ) and a given center of mass energy is the centrality of the collision. Considerable effort has been put by the ALICE Collaboration into an accurate determination of centrality using several detectors: final results for $\mathrm{Pb}-\mathrm{Pb}$ collisions are reported in [3]. The best centrality resolution for events with a good vertex determination has been obtained combining the signal amplitude of VZERO scintillator hodoscopes from both sides of the collision point: the resolution ranges from about $0.5 \%$ for very central (centrality near $0 \%$ ) events to about $2 \%$ for very peripheral (centrality approaching $80 \%$ ) events. Other methods use the Silicon Pixel Detector (SPD, the two innermost layers of the ITS) and the ZDC's combined with auxiliary e.m. calorimeters: the latter method is also suitable for collisions outside the main interaction region for centralities from $0 \%$ to $30 \%$.

More recently the methods of centrality determination have been extended to $\mathrm{p}-\mathrm{Pb}$ collisions. An example of centrality intervals determined using the VZERO-A ( $\mathrm{Pb}-$ going side) amplitude is shown in figure 10, where the amplitude spectrum has been fitted with a Glauber model including a Negative Binomal Distribution to represent the charged particle multiplicity generated in the nucleon-nucleon collisions. However using a centrality estimator based on multiplicity represents a bias in $\mathrm{p}-\mathrm{Pb}$ collisions (unlike the case of $\mathrm{Pb}-\mathrm{Pb}$ collisions); to avoid this bias a determination based on the ZDC as shown in figure 11 is very effective.

Having established a reliable centrality estimator, the first global observable whenever a new collision system or a new energy is explored is the charged particle multiplicity. For the 0-5\% most central $\mathrm{Pb}-\mathrm{Pb}$ collisions at $\sqrt{s_{\mathrm{NN}}}=2.76 \mathrm{TeV}$ ALICE has measured a charged particle multiplicity [4] of $1584 \pm 4$ (stat.) \pm 76 (syst.). This represents an increase of a factor 2.2 with respect to the top RHIC energy $(0.2 \mathrm{TeV})$; normalizing the charged particle yield to the number of pairs of participants and comparing the 5\% most central A-A collisions to pp collisions [4] we note that 


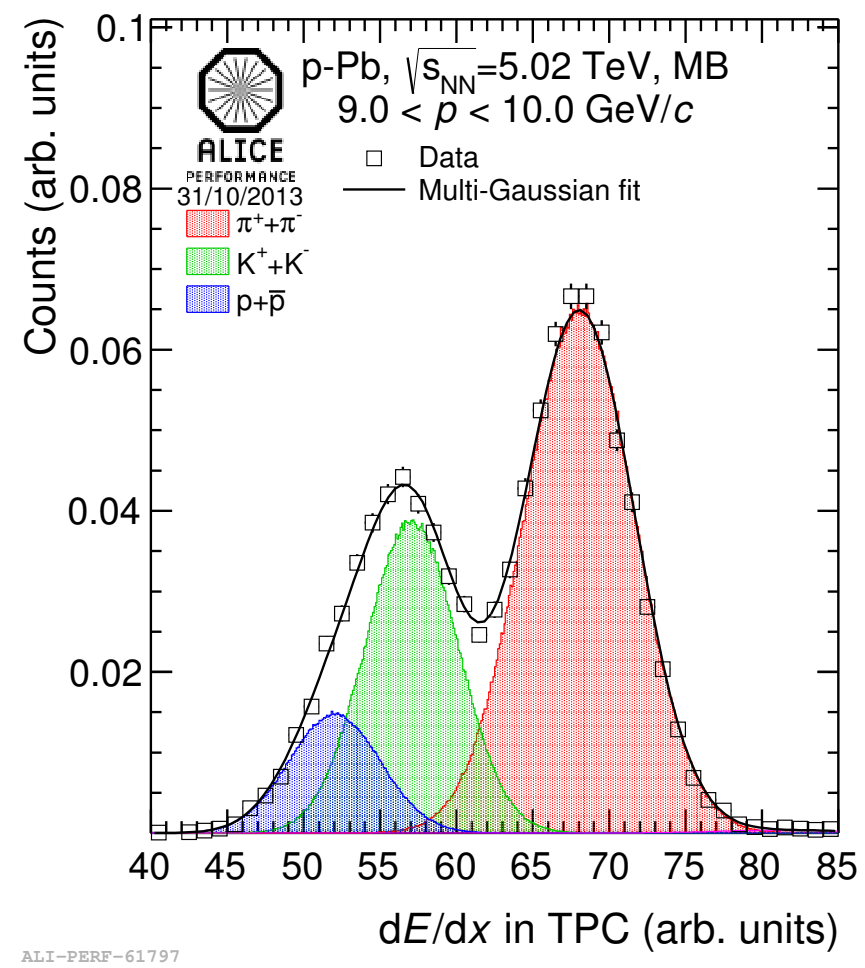

Figure 5: Separation between pions, kaons and protons on a statistical basis in the relativistic rise region of the TPC.

the yield per participant pair rises faster with $\sqrt{s}$ in central A-A collisions and that at the present LHC energy it is 1.9 times larger than in pp.

Results from ALICE on the charged particle pseudorapidity distributions in $\mathrm{Pb}-\mathrm{Pb}$ vs. centrality have been published in $[5,6]$. Combining measurements from central barrel detectors and forward detectors, $\mathrm{d} N_{\mathrm{ch}} / \mathrm{d} \eta$ has been obtained over more than 10 units of rapidity, allowing us to evaluate the total charged particle yield $N_{\text {ch }}$ virtually without extrapolation [6]; we have determined that the linear trend vs. $\ln ^{2} s_{\mathrm{NN}}$ valid from AGS to RHIC energies breaks down at LHC energy.

The charged particle yield vs. centrality in $\mathrm{Pb}-\mathrm{Pb}$ collisions has been presented and compared to models and RHIC results in [5]: the same shape vs. $N_{\text {part }}$ at RHIC has been observed at LHC, but with a scaling factor of 2.1. Among two-component models (perturbative QCD plus soft interactions) only HIJING 2.0 tuned to high energy pp data and central $\mathrm{Pb}-\mathrm{Pb}$ data was able to reproduce the increase of the yield vs. centrality, while models including gluon saturation were able to reproduce the centrality dependence and in one case the absolute yield.

More recently the results on $\mathrm{d} N_{\mathrm{ch}} / \mathrm{d} \eta$ in p- $\mathrm{Pb}$ collisions at $5.02 \mathrm{TeV}$ have been published [7]. For non-single-diffractive (NSD) interactions the measured value in $|\eta|<2$ is $16.95 \pm 0.75$; the shape of the $\mathrm{d} N_{\mathrm{ch}} / \mathrm{d} \eta$ distribution, which is important to assess initial state effects (the so-called cold nuclear matter, CNM), has been measured as well. Models that include gluon shadowing as HIJING 2.1 (having its gluon shadowing tuned to RHIC results) are close to ALICE data, while gluon saturation models show a steeper distribution than ALICE data. 


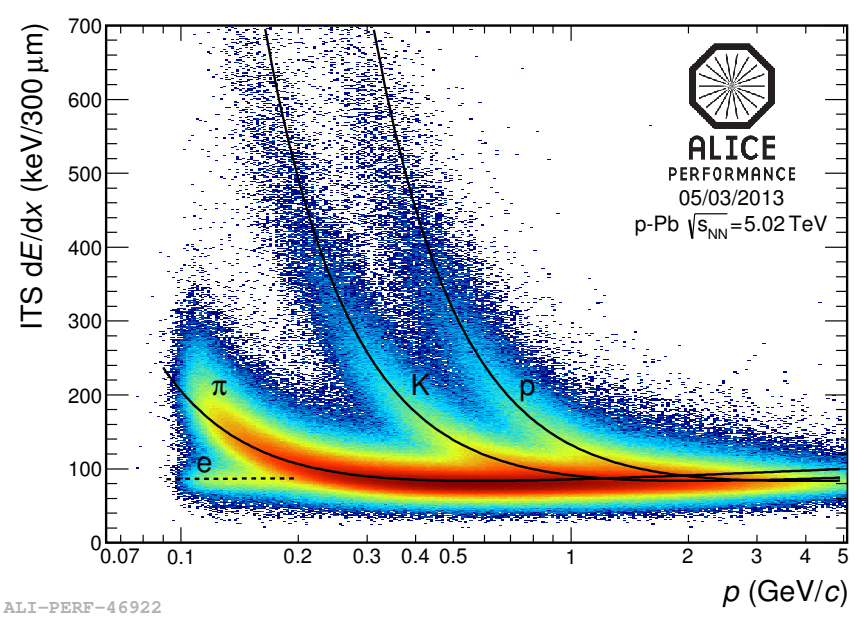

Figure 6: $\mathrm{d} E / \mathrm{d} x$ vs. momentum from the Internal Tracking System in $\mathrm{p}-\mathrm{Pb}$ collisions.

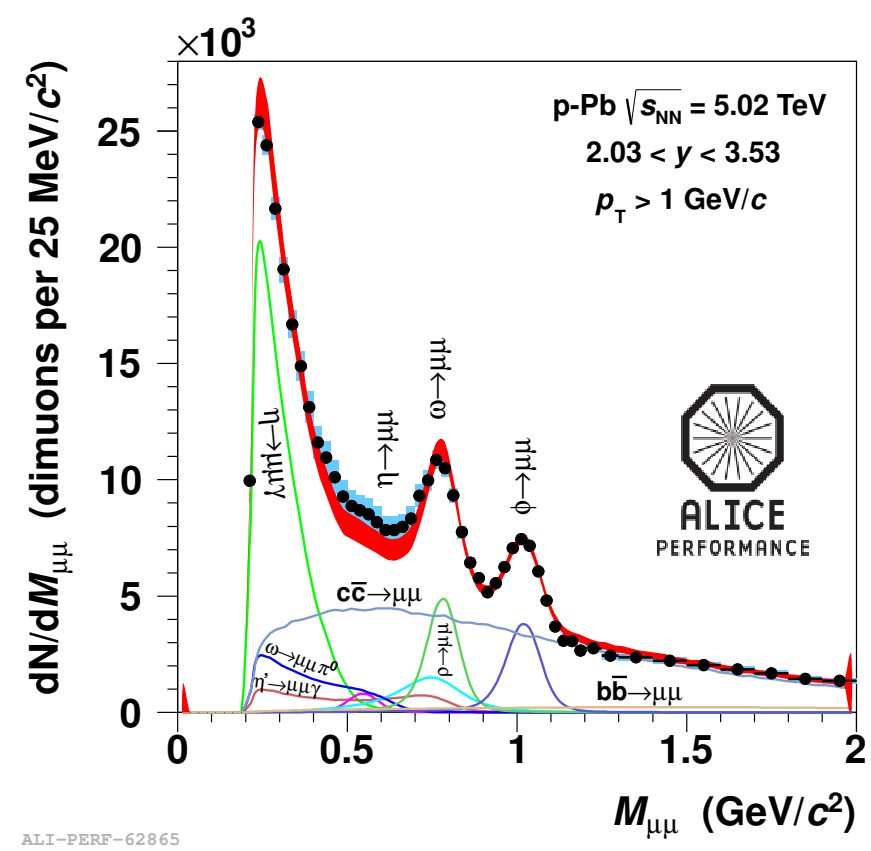

Figure 7: Dimuon invariant mass spectrum in the low-mass region in $\mathrm{p}-\mathrm{Pb}$ collisions.

The space-time volume of the homogeneity region has been determined mainly via identical pion interferometry. Early results [8] obtained at $\mathrm{LHC}$ in $\mathrm{Pb}-\mathrm{Pb}$ collisions indicated that the freezeout volume is about twice the one at RHIC, while the lifetime is larger by a factor about 1.4. More recently a thorough comparison of source radii between $\mathrm{pp}, \mathrm{p}-\mathrm{Pb}$ and $\mathrm{Pb}-\mathrm{Pb}$ colliding systems has been performed [9], with the aim of gathering information on the relative importance of initial conditions (which would favour $\mathrm{p}-\mathrm{Pb}$ radii similar to $\mathrm{pp}$ ) vs. hydrodynamics (which would favour larger radii in $\mathrm{p}-\mathrm{Pb}$, closer to $\mathrm{Pb}-\mathrm{Pb}$ ). For the same charged multiplicity, $\mathrm{p}-\mathrm{Pb}$ radii are 5-15\% larger than $\mathrm{pp}$, while $\mathrm{Pb}-\mathrm{Pb}$ radii are $35-45 \%$ larger than $\mathrm{p}-\mathrm{Pb}$. While $\mathrm{pp}$ and $\mathrm{p}-\mathrm{Pb}$ 


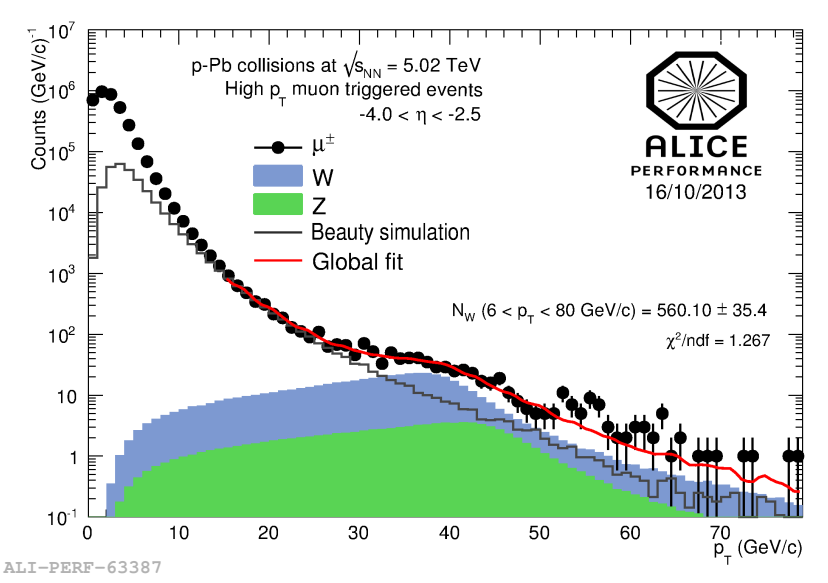

Figure 8: Single muon $p_{\mathrm{T}}$ spectrum in $\mathrm{p}-\mathrm{Pb}$ collisions.

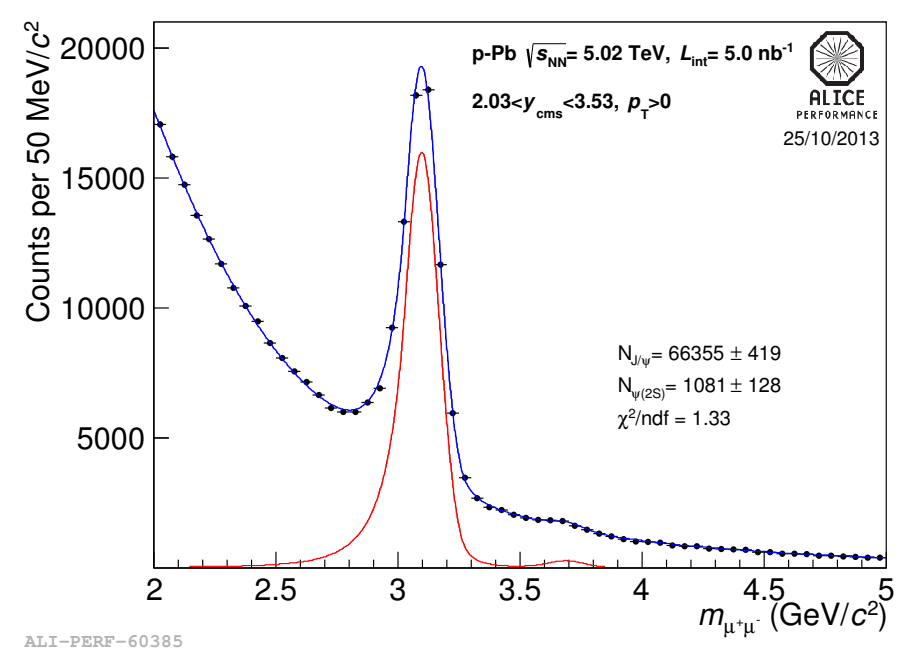

Figure 9: Dimuon invariant mass spectrum in the $J / \psi$ region in $\mathrm{p}-\mathrm{Pb}$ collisions.

radii can be reproduced by initial conditions including gluon saturation, those for $\mathrm{Pb}-\mathrm{Pb}$ require a hydrodynamical phase.

Another important indication on the global features of the collision comes from the direct photon $p_{\mathrm{T}}$ spectrum. A fit to preliminary ALICE data shown in figure 12 for $0-40 \%$ centrality $\mathrm{Pb}-\mathrm{Pb}$ collisions gives an inverse slope of $304 \pm 51 \mathrm{MeV}$ (about 1.4 larger than the result of the PHENIX experiment in 0-20\% Au-Au collisions at RHIC), which indicates initial temperatures between 500 and $600 \mathrm{MeV}$.

Finally, the average $p_{\mathrm{T}}$ vs. charged multiplicity has been studied [10] for the three colliding systems. We have observed that $\mathrm{p}-\mathrm{Pb}$ and even more pp systems show a much stronger increase of the average transverse momentum vs. $N_{\mathrm{ch}}$ than $\mathrm{Pb}-\mathrm{Pb}$. The increase in pp data is reasonably well described by PYTHIA 8 (tune 4C) but only when color reconnection is turned on. A simple Glauber model superposition of $\mathrm{pp}$ and $\mathrm{pn}$ collisions fails to describe $\mathrm{p}-\mathrm{Pb}$ and $\mathrm{Pb}-\mathrm{Pb}$ data; no model among DPMJET, HIJING, AMPT and EPOS is able to describe $\mathrm{p}-\mathrm{Pb}$ and $\mathrm{Pb}-\mathrm{Pb}$ data consistently, 


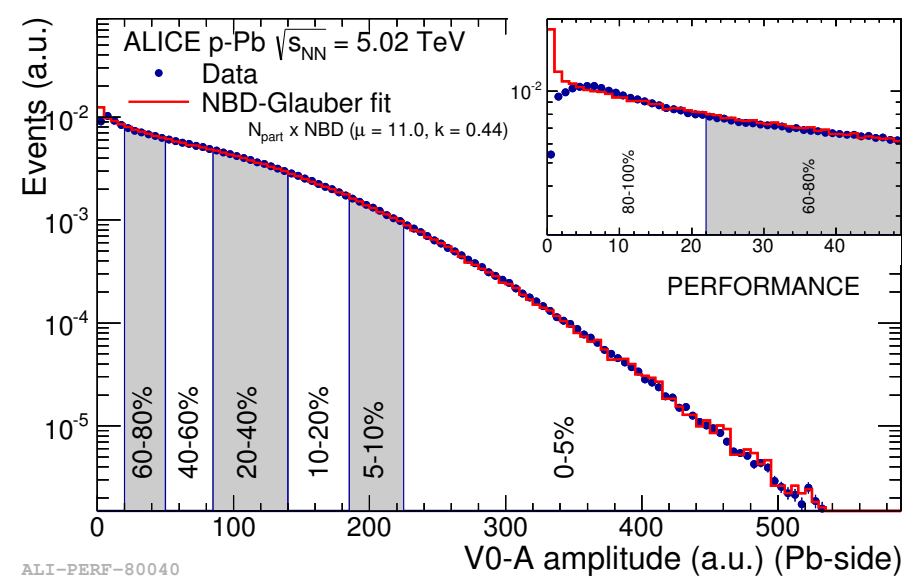

Figure 10: Centrality classes in $\mathrm{p}-\mathrm{Pb}$ as determined from the V0-A scintillator array.

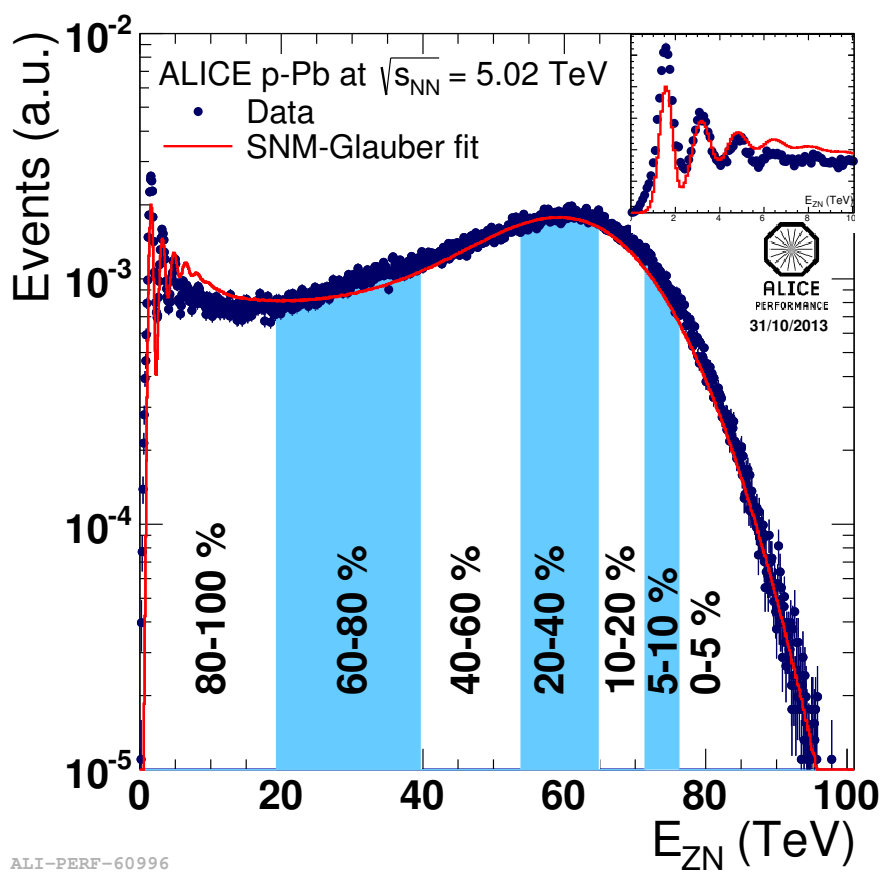

Figure 11: Centrality classes in $\mathrm{p}-\mathrm{Pb}$ as determined from the neutron ZDC energy.

however EPOS is close to $\mathrm{p}-\mathrm{Pb}$ data.

\section{Particle production}

Thanks to the excellent performance of particle identification in the central barrel (ITS, TPC, TOF and HMPID), transverse momentum spectra of identified hadrons in a wide range have been obtained for $\mathrm{pp}, \mathrm{p}-\mathrm{Pb}$ and $\mathrm{Pb}-\mathrm{Pb}$ systems. These spectra represent the input data for the flavour and mass dependences of particle yields, as well as for the study of the evolution of yields with colliding system and centrality. 


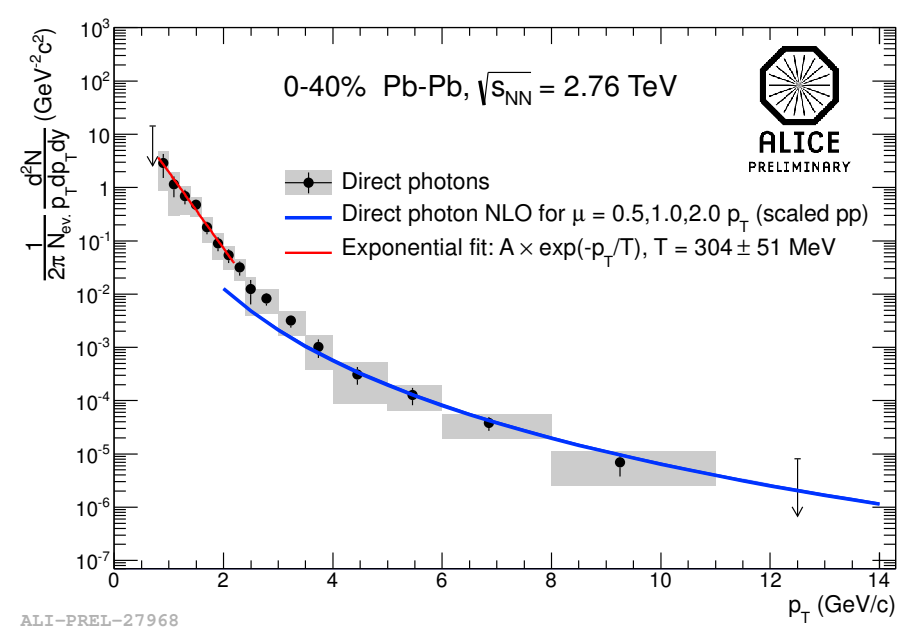

Figure 12: Direct photon $p_{\mathrm{T}}$ spectrum in $\mathrm{Pb}-\mathrm{Pb}$ collisions.

ALICE results on identified charged particle production in pp collisions and for two centrality classes in $\mathrm{Pb}-\mathrm{Pb}$ collisions have been published in [11], while results on the multiplicity dependence of the production in $\mathrm{p}-\mathrm{Pb}$ collisions are reported in [12].

Hadron spectra in $0-5 \%$ central $\mathrm{Pb}-\mathrm{Pb}$ collisions have been presented and compared to several models in [13]. ALICE data are well described by several hydrodynamical models. A blast-wave fit to the spectra was performed to extract the kinetic freeze-out temperature and the radial flow velocity: a kinetic temperature of about $95 \mathrm{MeV}$ (similar to RHIC) was obtained, together with a radial flow velocity of $0.65 c, 10 \%$ larger than at RHIC.

The so called baryon anomaly, namely an enhancement in the proton/pion and $\Lambda / K_{s}^{0}$ ratios at intermediate $p_{\mathrm{T}}$, has been observed in A-A collisions at RHIC and confirmed at LHC. In particular, the $\Lambda$ production is strongly enhanced with respect to $K_{s}^{0}$ in central $\mathrm{Pb}-\mathrm{Pb}$ collisions (see [14]). The same ratio in inclusive $\mathrm{p}-\mathrm{Pb}$ collisions is similar to that observed in peripheral $\mathrm{Pb}-\mathrm{Pb}$ collisions, while no enhancement has been found in jets, as shown in figure 13.

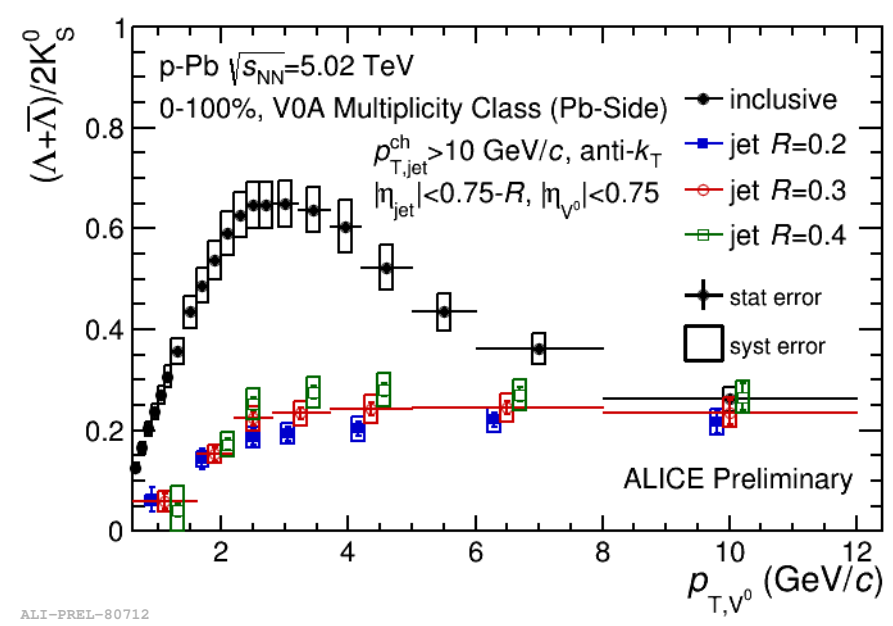

Figure 13: Strange baryon/meson ratio vs. $p_{\mathrm{T}}$ in $\mathrm{p}-\mathrm{Pb}$ collisions. 
Particle yield ratios have been studied systematically vs. energy in pp collisions and for different systems at LHC top energy. Figure 14 shows yield ratios among several hadrons (including light nuclei) in pp collisions at three different energies: a weak (if any) energy dependence is present in the range $0.9-7 \mathrm{TeV}$.
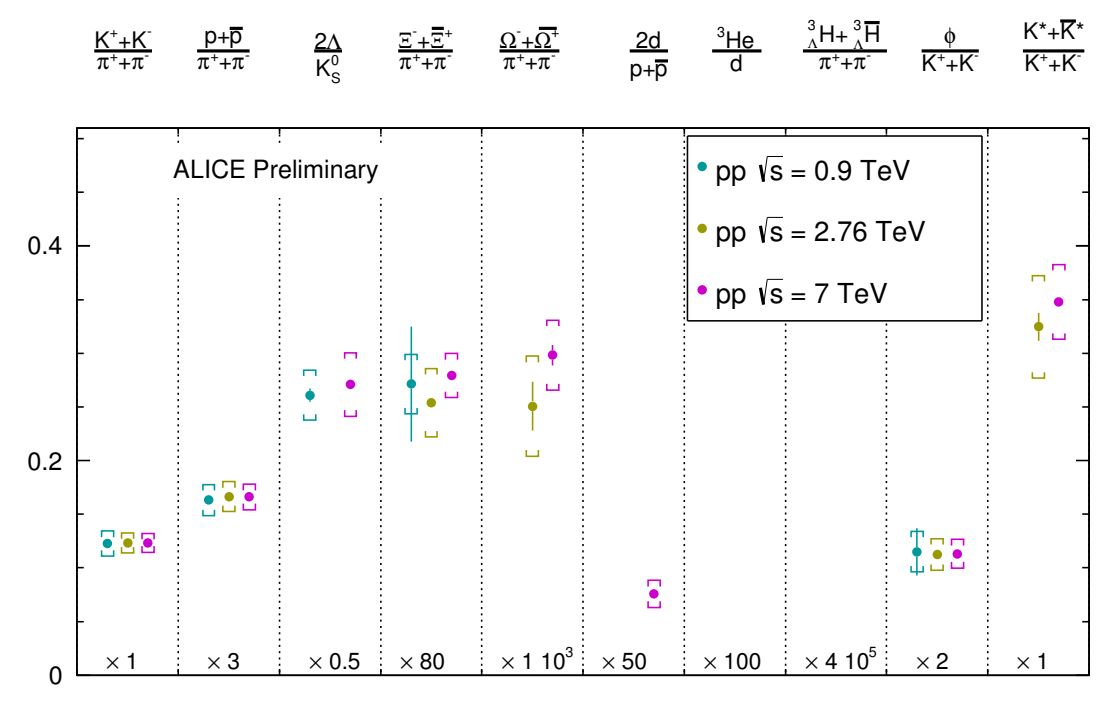

ALI-PREL -74045

Figure 14: Energy dependence of particle yield ratios in pp collisions.

The comparison of yields at top LHC energy among pp, 0-5\% multiplicity $\mathrm{p}-\mathrm{Pb}$ and $0-10 \%$ centrality $\mathrm{Pb}-\mathrm{Pb}$ collisions is presented in figure 15 . Yield ratios appear to be quite similar among different systems, however in central $\mathrm{Pb}-\mathrm{Pb}$ an enhancement of the $\Omega$ and a suppression of $\mathrm{p}$ and $K^{*}$ is apparent with respect to $\mathrm{pp}$ and $\mathrm{p}-\mathrm{Pb}$. The enhancement of multi-strange baryon yields in central $\mathrm{Pb}-\mathrm{Pb}$ collisions is one of the early signatures of the QGP, and is currently attributed to a reduced influence of canonical suppression of strangeness in larger volumes.

The absolute yields $(\mathrm{d} N / \mathrm{d} y)$ in central $\mathrm{Pb}-\mathrm{Pb}$ collisions are compared in figure 16 to equilibrium models including THERMUS [15], GSI [16] and SHARE [17]. All thermal models provide a rather good description of ALICE data with two parameters, namely the temperature and the volume of the fireball, with the notable exception of protons where models are above data by more than 2 standard deviations.

\section{Collective effects}

Collective effects in A-A collisions are observable through momentum correlations among produced particles; in particular non-central collisions are characterized by an initial space anisotropy which is converted into an anisotropy in momentum space for the final hadrons. The anisotropic flow coefficients $v_{n}$ are extracted (as a function of $p_{\mathrm{T}}$ ) with a Fourier expansion of the azimuthal distribution in the transverse plane; several methods (including event plane and cumulants) are used in order to understand and subtract the non-flow contribution to the $v_{n}$ coefficients.

The charged particle elliptic flow coefficient $\left(v_{2}\right)$ for different centrality classes in $\mathrm{Pb}-\mathrm{Pb}$ collisions at LHC has been reported in [18]. While the functional form of $v_{2}\left(p_{\mathrm{T}}\right)$ for charged particles 


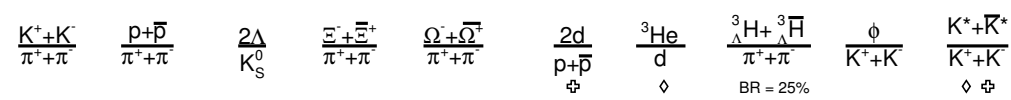

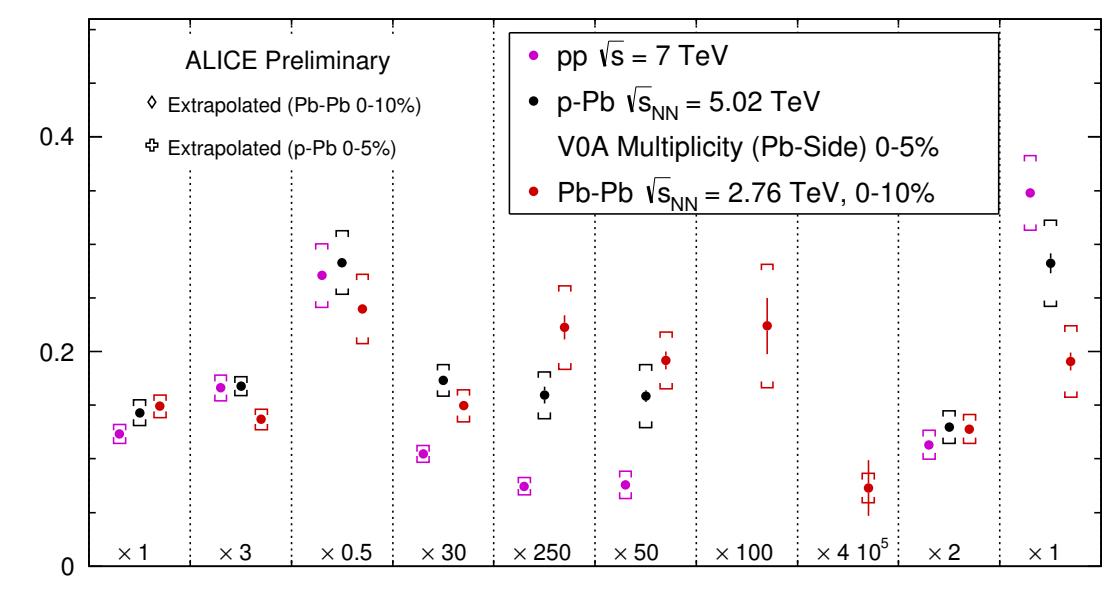

ALI-PREL -74423

Figure 15: Particle ratios in pp collisions and in central $\mathrm{p}-\mathrm{Pb}$ and $\mathrm{Pb}-\mathrm{Pb}$ collisions at top LHC energy.

does not change going from RHIC to LHC energies, the $p_{\mathrm{T}}$-integrated value is larger at LHC, due to the larger average $p_{\mathrm{T}}$.

Higher harmonic coefficients (up to $v_{5}$ ) have been reported in [19] and [20] for charged particles in $\mathrm{Pb}-\mathrm{Pb}$ collisions. Fourier components with $n>2$ arise due to fluctuations in the initial geometry; they are extracted either via the correlation of produced particles with the $n$-th symmetry plane or via the correlation between a trigger and an associate particle, separated by a pseudorapidity gap (typically of 0.8 units). The set of $v_{n}$ measurements provides crucial information for the extraction of the ratio of bulk viscosity to entropy density in hydrodynamical models.

More recently, the elliptic flow coefficients of identified hadrons measured by ALICE in $\mathrm{Pb}-$ $\mathrm{Pb}$ collisions have been published [21]. Charged stable hadrons, neutral kaons, strange baryons and the $\phi$ meson have been measured; mass ordering for $v_{2}$ has been observed, as expected from a common radial expansion velocity. The $\phi$ meson is behaving more like a baryon, meaning that its mass is more relevant than its quark composition. The scaling of $v_{2}$ with the number of constituent quarks is less obvious at LHC in comparison to RHIC.

Still concerning particle correlations, in $\mathrm{p}-\mathrm{Pb}$ collisions at high multiplicity ALICE has observed a double ridge in the azimuthal distribution, see [22]. The interpretation of this observation is still open, since both the Color Glass Condensate (initial state effect) and hydrodynamics (final state effect) are able to reproduce it.

\section{Jet quenching}

The modification of jets in the hot QCD medium is a key observable, as first established at RHIC. ALICE is capable of measuring both charged jets in the barrel $(|\eta|<0.9$ and full azimuth) and the electromagnetic component in the EMCal $\left(|\eta|<0.3\right.$ and $\frac{1}{3}$ azimuth).

The full (charged plus neutral components) jet $p_{\mathrm{T}}$ spectrum for $0-10 \%$ centrality $\mathrm{Pb}-\mathrm{Pb}$ collisions is shown in figure 17. Using pp reference data, the nuclear modification factor $R_{\mathrm{AA}}$ as a 


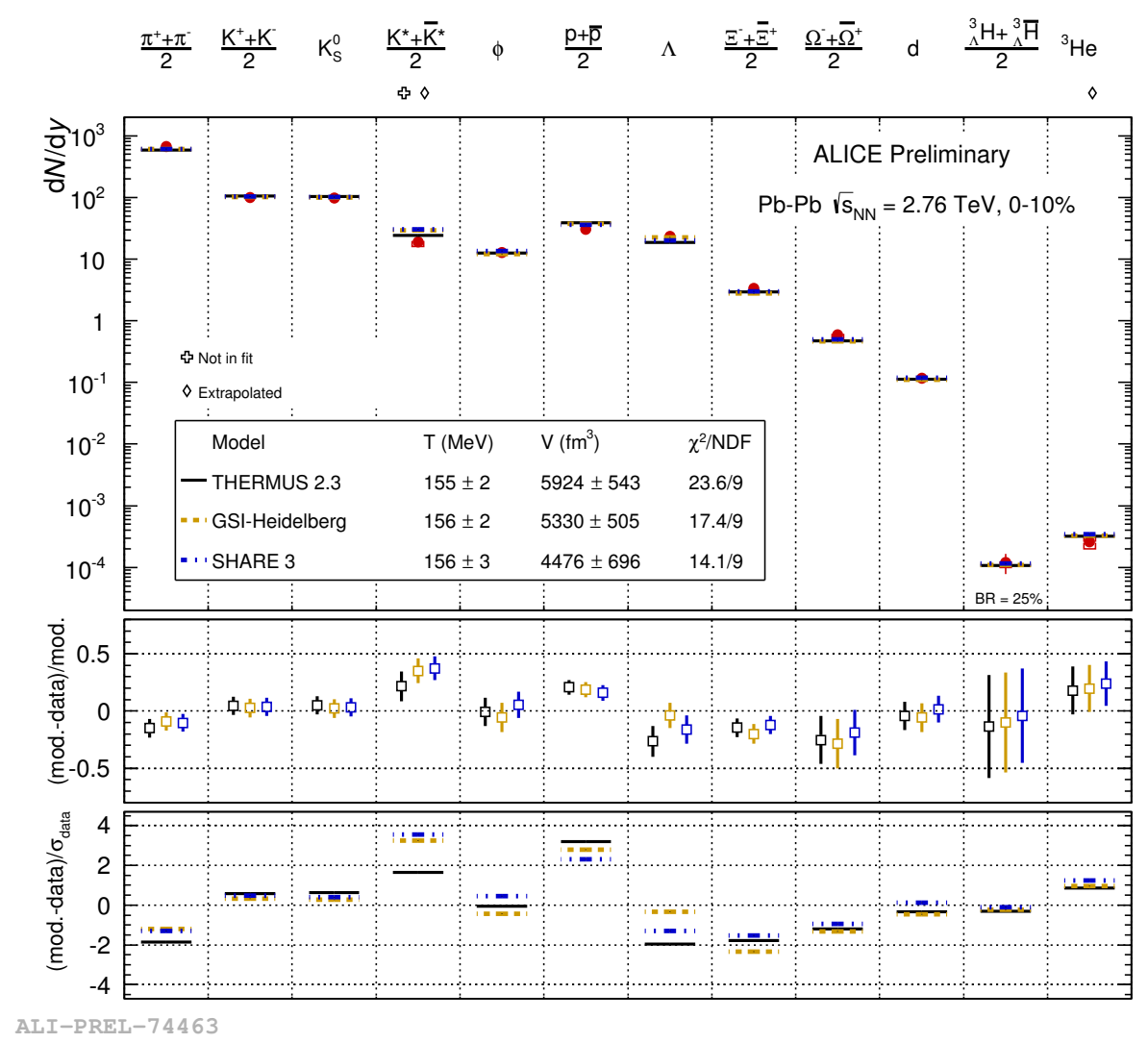

Figure 16: Particle ratios in central $\mathrm{Pb}-\mathrm{Pb}$ collisions compared to thermal models.

function of $p_{\mathrm{T}}$ is shown in figure 18: a clear suppression $\left(R_{\mathrm{AA}}\right.$ between 0.25 and 0.5$)$ is observed in the $30-120 \mathrm{GeV} / c p_{\mathrm{T}}$ range.

The nuclear modification factor for charged jets in $\mathrm{p}-\mathrm{Pb}$ collisions is shown in figure 19: no modification relative to pp collisions is observed, meaning that there is no significant cold nuclear matter effect on jets.

Charged particle suppression in $\mathrm{Pb}-\mathrm{Pb}$ collisions has been extensively studied by ALICE, both in central collisions [23] and as a function of centrality [24]. A strong suppression of charged particles in central $\mathrm{Pb}-\mathrm{Pb}$ collisions has been observed up to $50 \mathrm{GeV} / c$, roughly matching the one observed for jets (although one must bear in mind the different meaning of $p_{\mathrm{T}}$ for the leading charged particle and for the jet).

The measurement of the charged particle nuclear modification factor in $\mathrm{p}-\mathrm{Pb}$ collisions has been reported up to $20 \mathrm{GeV} / \mathrm{c}$ in [25] and it has been recently extended up to $50 \mathrm{GeV} / \mathrm{c}$ in [26]. The $\mathrm{p}-\mathrm{Pb}$ results confirm that the strong suppression seen in $\mathrm{Pb}-\mathrm{Pb}$ is due to hot nuclear matter effects $\left(R_{\mathrm{pA}}\right.$ is consistent with unity up to the highest $\left.p_{\mathrm{T}}\right)$.

More recently, the nuclear modification factor for identified hadrons in $\mathrm{p}-\mathrm{Pb}$ collisions has been studied in ALICE: some results are shown in figures 20 (pions), 21 (kaons), 22 (protons and antiprotons) and 23 ( $\phi$ mesons).

While confirming that $R_{\mathrm{pPb}}$ approaches unity at high $p_{\mathrm{T}}$ for all measured species, one observes 


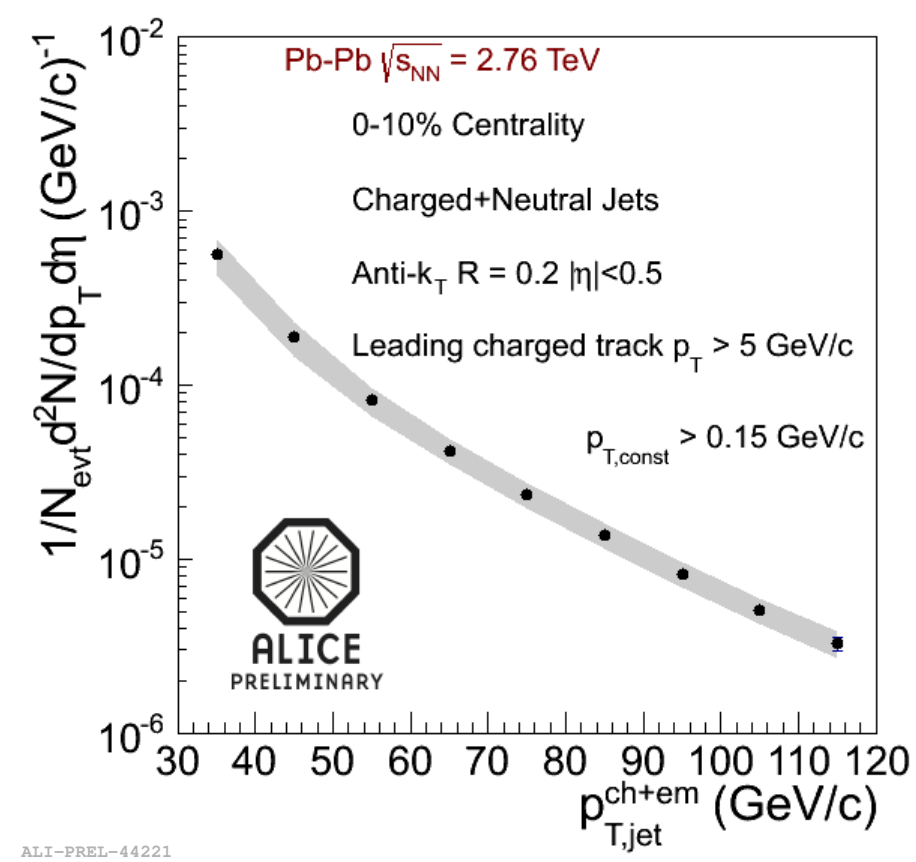

Figure 17: Full jet $p_{\mathrm{T}}$ spectrum in central $\mathrm{Pb}-\mathrm{Pb}$ collisions.

a Cronin enhancement for baryons in the 3-5 GeV/c transverse momentum range; on the other hand, the $\phi$ meson nuclear modification factor is closer to the one of pions and kaons. 


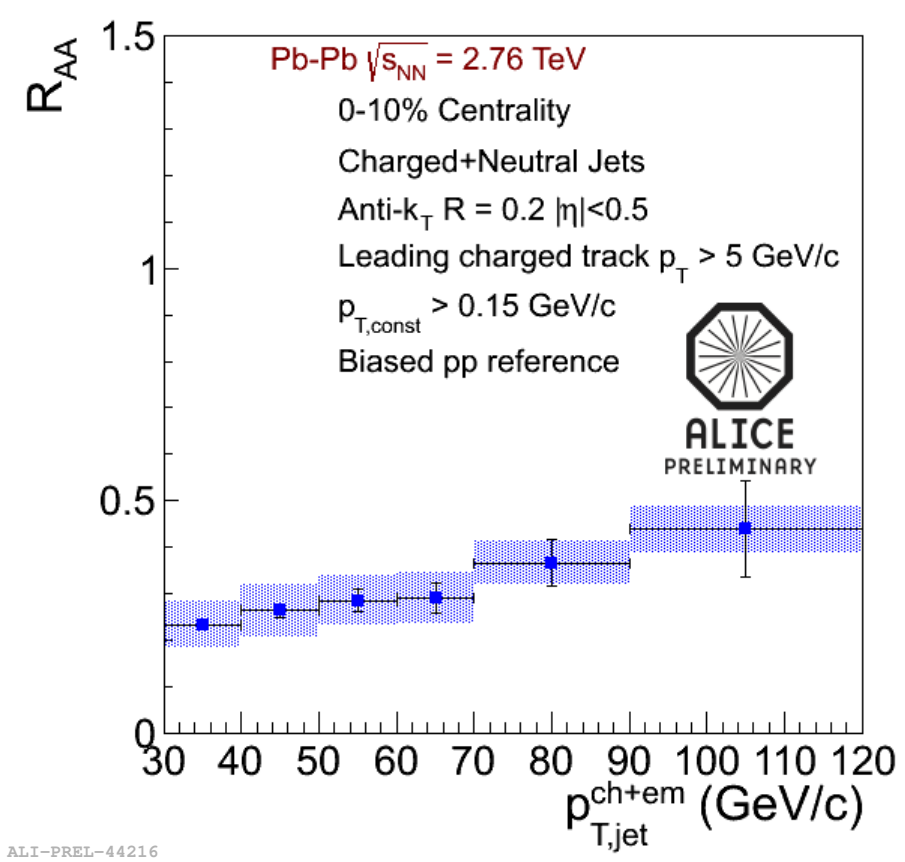

Figure 18: Full jet nuclear suppression factor vs. $p_{\mathrm{T}}$ in central $\mathrm{Pb}-\mathrm{Pb}$ collisions.

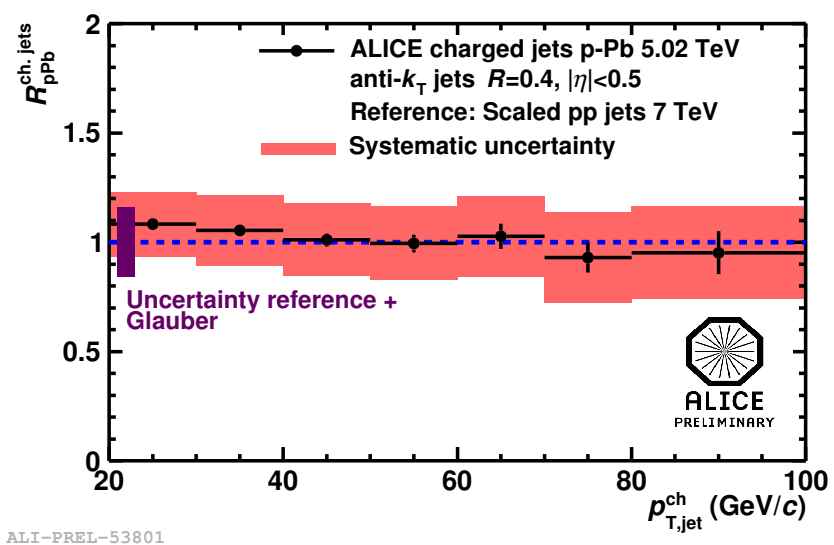

Figure 19: Nuclear modification factor vs. $p_{\mathrm{T}}$ for charged jets in $\mathrm{p}-\mathrm{Pb}$ collisions. 


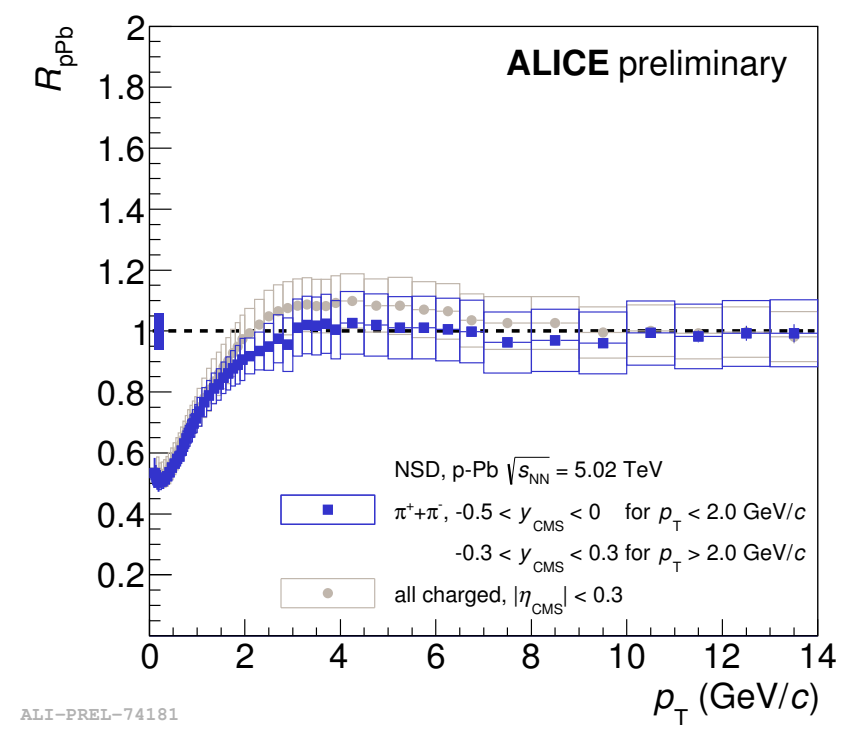

Figure 20: Nuclear modification factor for charged pions in $\mathrm{p}-\mathrm{Pb}$ collisions.

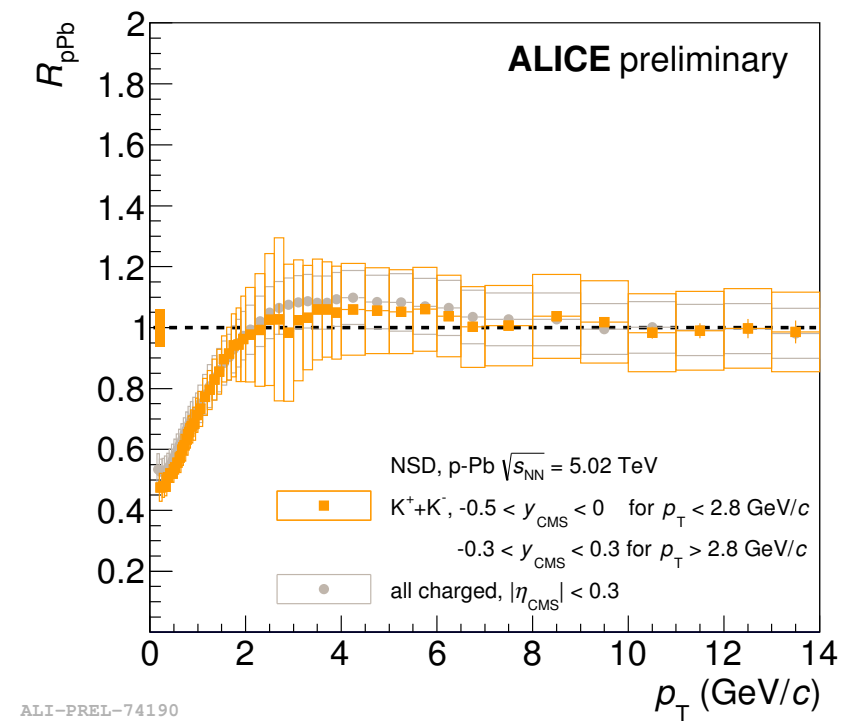

Figure 21: Nuclear modification factor for charged kaons in $\mathrm{p}-\mathrm{Pb}$ collisions. 


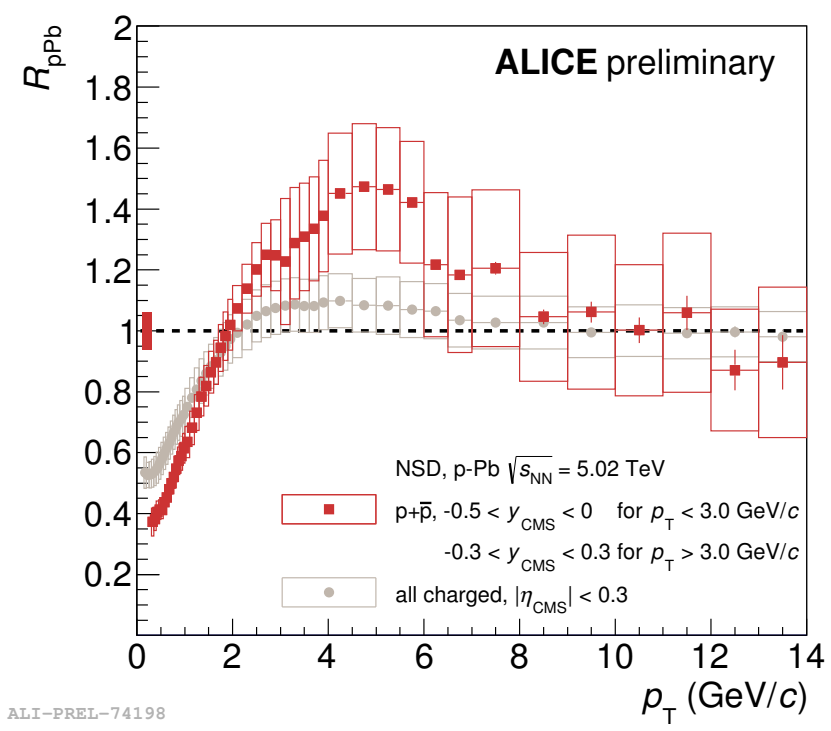

Figure 22: Nuclear modification factor for (anti)protons in $\mathrm{p}-\mathrm{Pb}$ collisions.

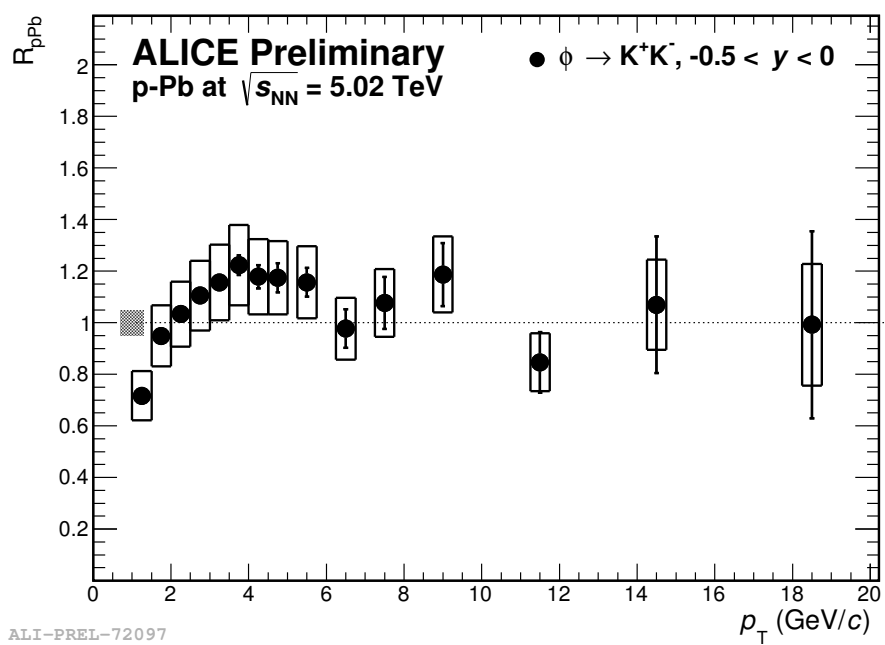

Figure 23: Nuclear modification factor for $\phi$ mesons in $\mathrm{p}-\mathrm{Pb}$ collisions. 


\section{Open heavy flavours}

Heavy quarks provide another very important tool to probe the QCD matter created in A-A collisions (see e.g. [27]), since they are produced in the initial hard scattering and then experience the full system evolution; their number being conserved, they are indeed a unique tool to characterize the medium, in particular via their energy loss.

In ALICE, heavy flavours (charm and beauty) are identified via fully reconstructed decay modes or via semileptonic decays. Suppression of $\mathrm{D}-$ mesons in $0-20 \%$ centrality $\mathrm{Pb}-\mathrm{Pb}$ collisions has been reported in [28]. Recent results in the 0-7.5\% centrality range are shown in figure 24, where the nuclear modification factor of four different D-meson species is reported. A strong suppression of prompt D-mesons is observed, up to a factor 5 for $p_{\mathrm{T}}$ of about $10 \mathrm{GeV} / c$; there is furthermore a hint of a higher $R_{\mathrm{AA}}$ for the $D_{s}^{+}$meson at low $p_{\mathrm{T}}$.

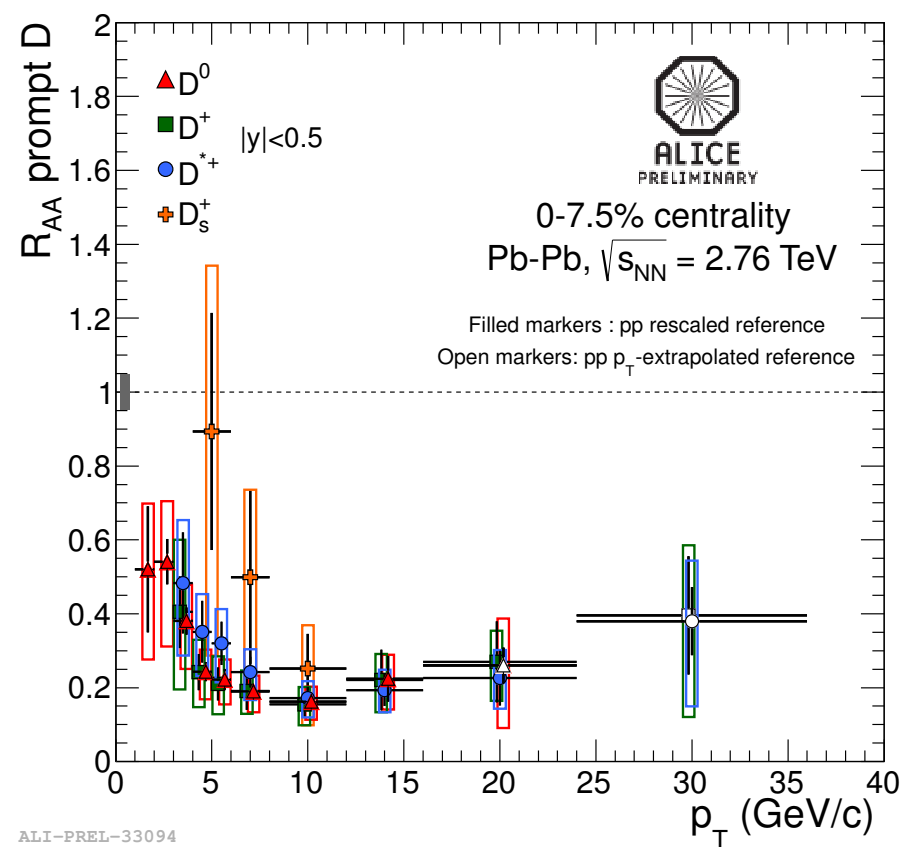

Figure 24: Nuclear modification factor for prompt $\mathrm{D}$ mesons in central $\mathrm{Pb}-\mathrm{Pb}$ collisions.

The measurement of the nuclear modification factor of $\mathrm{D}-$-mesons in $\mathrm{p}-\mathrm{Pb}$ collisions has recently been published (see [29]), confirming that cold nuclear matter effects are small and that the suppression observed in central $\mathrm{Pb}-\mathrm{Pb}$ collisions is due to strong final-state effects induced by hot partonic matter.

In order to assess whether the mass ordering of energy loss between charm and beauty is indeed observed, a comparison between ALICE prompt D-mesons and CMS non-prompt $J / \psi$ from B decays (from [30]) is shown in figure 25 as a function of centrality. The expected mass ordering is indeed observed, and is reproduced by a pQCD model which includes mass dependent radiative and collisional energy loss, as shown in figure 26.

Very similar results to those shown for D and B mesons have been obtained by ALICE using leptons from heavy flavour decays, both in the central barrel (electrons and muons) and in the forward muon spectrometer. 


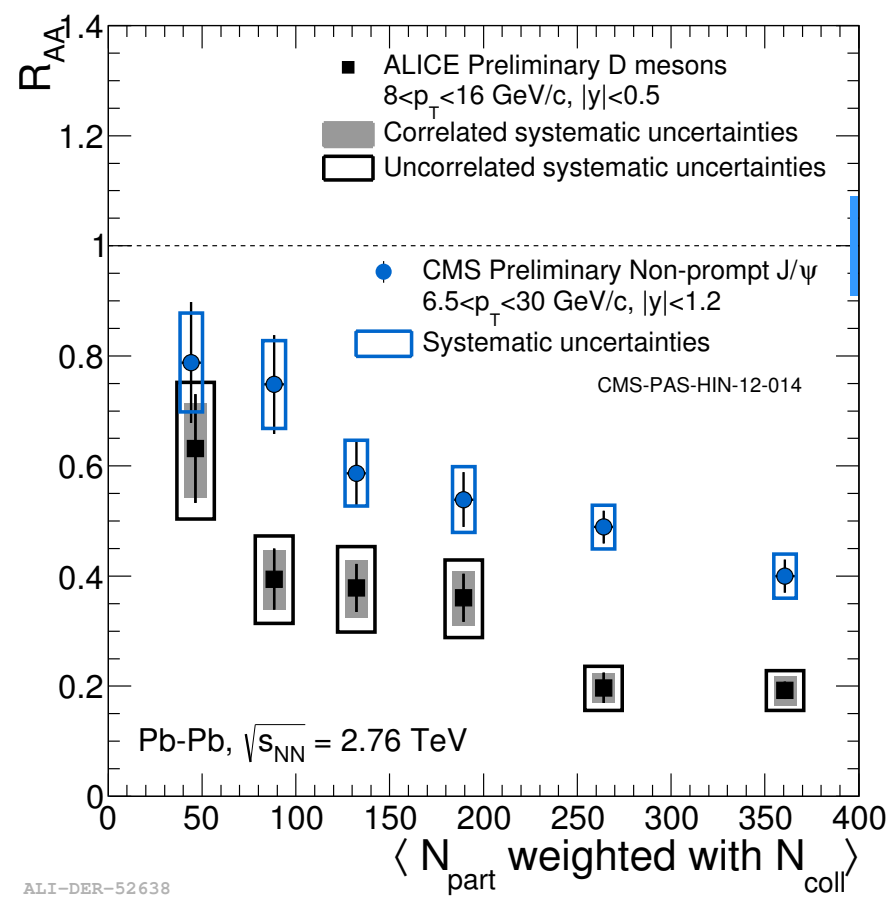

Figure 25: Comparison of nuclear modification factors between D mesons (ALICE) and $J / \psi$ from B decays (CMS ref. [30]).

Recently the $v_{2}$ (elliptic flow coefficient) of D-mesons has been measured (see [31]), being non-zero in semi-central $\mathrm{Pb}-\mathrm{Pb}$ collisions and similar to the charged particles one. This observation implies that there is a significant interaction of charm quarks with the medium, suggesting collective motion of low $p_{\mathrm{T}}$ charm quarks in the expanding fireball.

A comparison of the measured $v_{2}$ with several models is presented in figure 27: it is seen that the description of charm flow is challenging even for models which have been shown to describe reasonably well the nuclear modification factor $R_{\mathrm{AA}}$.

\section{Quarkonia}

Quarkonia have been identified as a crucial probe for the QGP temperature quite early [32]. Later on, the importance of sequential suppression of different bound states and the thermal regeneration mechanism have been clarified (see e.g. [33] and [34]). The colour screening in the QGP prevents the binding of heavy quark-antiquark pairs when the radius of the bound state is larger than the screening ratio, which in turn depends on the temperature. Detailed predictions on the survival probability of different charmonium and bottonium states vs. the ratio of the actual temperature to the critical temperature are given in [34]. At RHIC and even more at LHC energies, the regeneration (recombination) mechanism becomes important, due to the increase in heavy quark-antiquark pair abundance.

ALICE has recently published [35] results on the $J / \psi$ nuclear modification factor vs. centrality in $\mathrm{Pb}-\mathrm{Pb}$ collisions, both at central and forward rapidities. A clear suppression is seen, with almost no centrality dependence for a number of participants larger than 100; on the other 


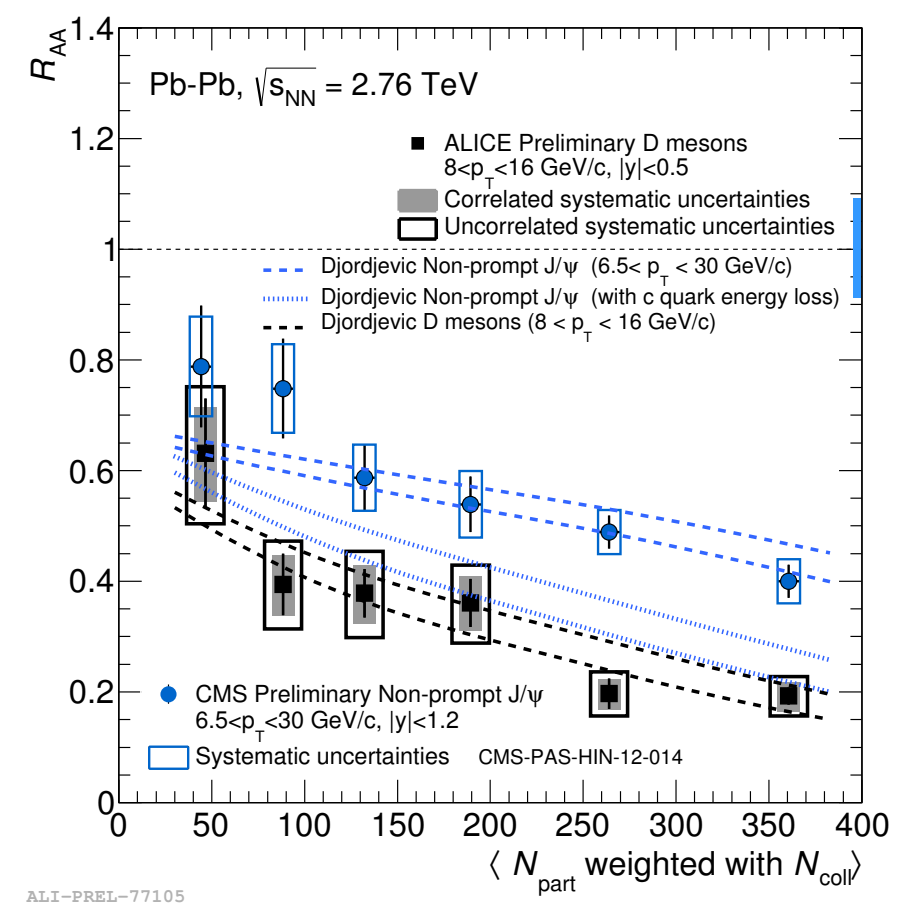

Figure 26: Comparison between D meson (ALICE), $J / \psi$ from B decays (CMS ref. [30]) and models nuclear modification factors.

hand, the suppression at mid-rapidity is less strong when compared to forward rapidity. A comparison with RHIC results [36] from the PHENIX experiment indicates that in ALICE at LHC energy the suppression has a weaker centrality dependence, and also that in central $\mathrm{Pb}-\mathrm{Pb}$ collisions the suppression is less strong: this observation points to a larger contribution from statistical recombination at LHC energy.

A crucial cross-check for the proposed explanation is the measurement of the cold nuclear matter (CNM) effects, due for example to nuclear shadowing of the gluon PDF, in $\mathrm{p}-\mathrm{Pb}$ collisions. $\mathrm{A}$ recent comparison of $\mathrm{p}-\mathrm{Pb}$ and $\mathrm{Pb}-\mathrm{Pb}$ results from ALICE is shown in figure 28: the nuclear modification factor $R_{\mathrm{pA}}$ is compatible with unity at backward rapidities and decreases toward forward rapidity, in agreement with shadowing and coherent energy loss models.

Using the measured $\mathrm{CNM}$ effect in $\mathrm{p}-\mathrm{Pb}$ and $\mathrm{Pb}-\mathrm{p}$ collisions, one can extrapolate the effect to the $\mathrm{Pb}-\mathrm{Pb}$ kinematical range and compare with the measured $R_{\mathrm{PbPb}}$, as shown in figure 29 . The comparison indicates that indeed hot matter effects are seen in $\mathrm{Pb}-\mathrm{Pb}$.

\section{Outlook}

The vast array of results obtained by ALICE with LHC Run 1 prompted the Collaboration to make ambitious plans for the upcoming Run 2 and to propose a major upgrade of the experiment for the projected Run 3 (2020-2022 and beyond).

For the data taking in Run 2 the energy will be up to $13 \mathrm{TeV}$ for pp collisions and about 5.1 $\mathrm{TeV}$ for $\mathrm{Pb}-\mathrm{Pb}$ collisions, so the main emphasis will be on the higher energy (about a factor 2 in both cases). ALICE plans to collect about $70 \mathrm{nb}^{-1}$ with minimum bias pp collisions, $10 \mathrm{pb}^{-1}$ with 


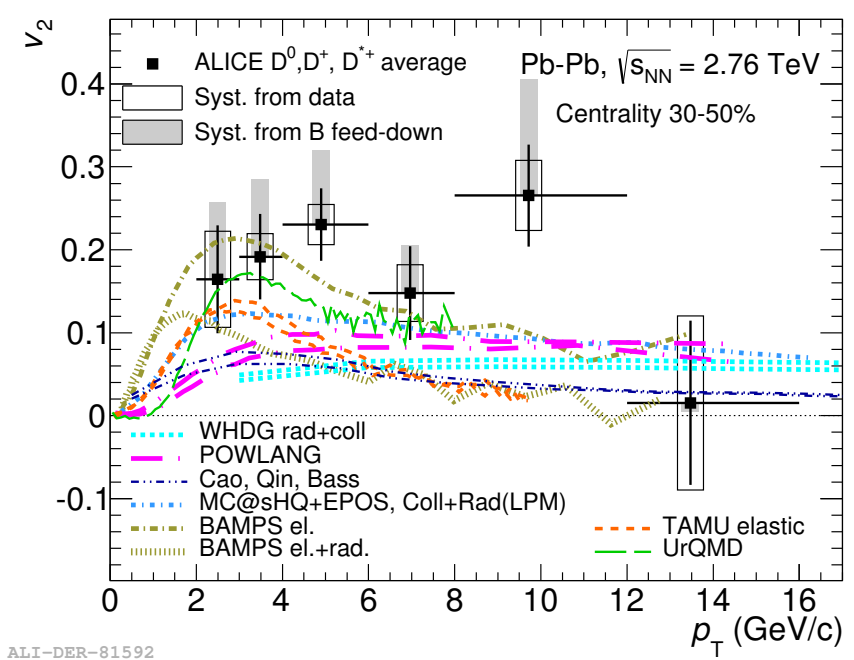

Figure 27: $\mathrm{D}$ meson elliptic flow coefficient $v_{2}$ vs. $p_{\mathrm{T}}$ in semicentral $\mathrm{Pb}-\mathrm{Pb}$ collisions.

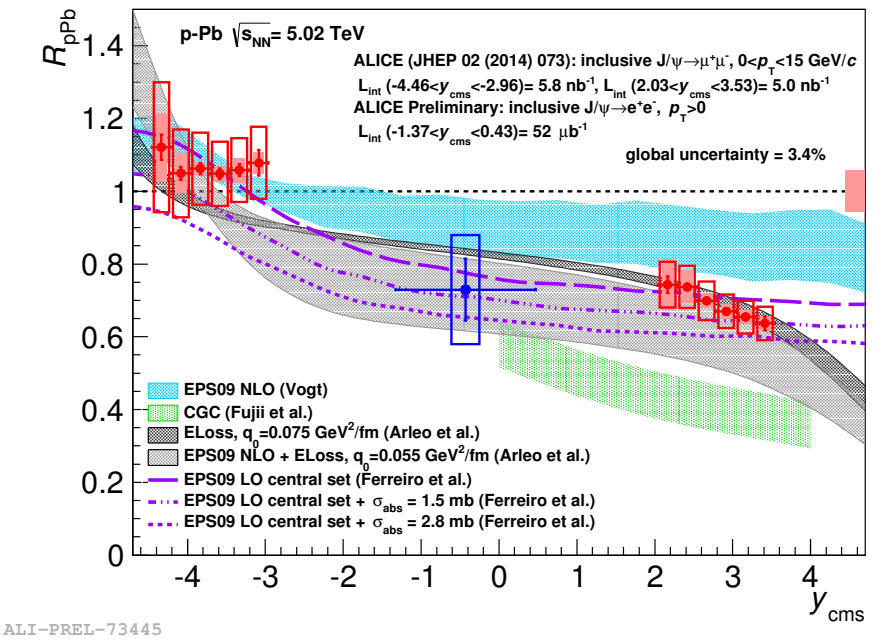

Figure 28: $J / \psi$ nuclear modification factor in $\mathrm{p}-\mathrm{Pb}$ collisions compared to models.

rare pp triggers and $1 \mathrm{nb}^{-1}$ with $\mathrm{Pb}-\mathrm{Pb}$ collisions. The geometrical coverage will be completed for a few detectors which were not fully ready in Run 1, and major upgrades will be made to the trigger and readout systems.

Major upgrades of the ITS, of the TPC readout chambers and the addition of the Muon Forward Tracker are being planned (see [37]) for Run 3, with main emphasis on the much higher luminosity. The improvement in vertexing capability and tracking at low $p_{\mathrm{T}}$ and the increase of the data taking capability of a factor 100 (up to at least $50 \mathrm{kHz}$ interaction rate in $\mathrm{Pb}-\mathrm{Pb}$ ) will allow for precision studies of charm and beauty mesons and baryons at low $p_{\mathrm{T}}$, a detailed study of low-mass lepton pairs and thermal photons, the study of $\gamma$-jet and dijets with particle identification and the study of heavier nuclear states. The advanced state of the ALICE upgrade R \& D and planning is well documented in [38]. 


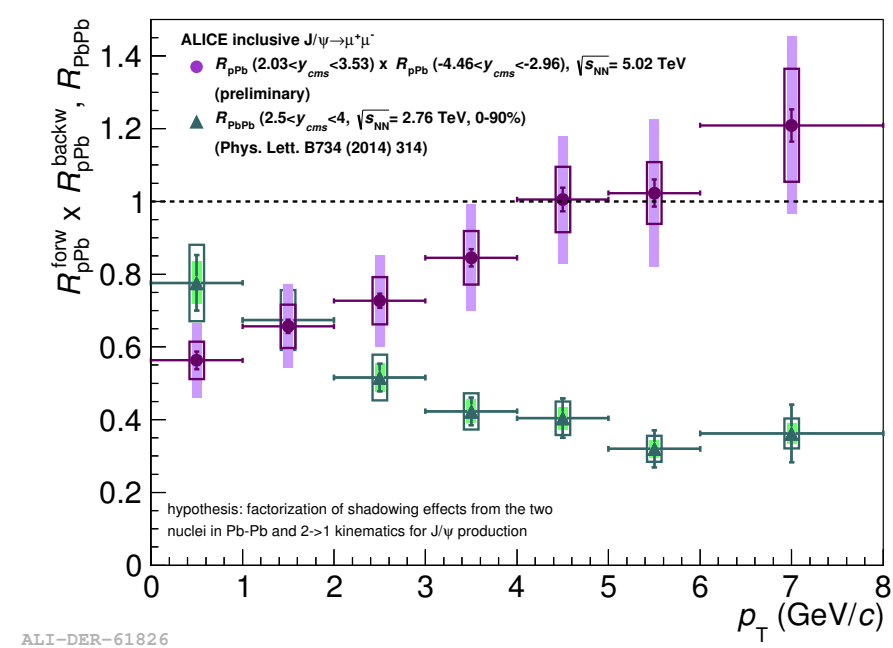

Figure 29: Nuclear modification factor vs. $p_{\mathrm{T}}$ for $J / \psi$ in $\mathrm{p}-\mathrm{Pb}$ and in $\mathrm{Pb}-\mathrm{Pb}$ collisions.

\section{References}

[1] J. Schukraft, The Future of High Energy Nuclear Physics in Europe, invited talk at the 'D. A. Bromley Memorial Symposium', Yale University, USA, 8-9 December 2005, nucl-ex/ 0602014

[2] The ALICE Collaboration, K. Aamodt et al., The ALICE Experiment at the CERN LHC, JINST 3 (2008) S08002

[3] ALICE Collaboration, B. Abelev et al., Centrality determination of $P b-P b$ collisions at $\sqrt{s_{\mathrm{NN}}}=2.76$ TeV with ALICE, Phys. Rev. C 88 (2013) 044909

[4] ALICE Collaboration, K. Aamodt et al., Charged-particle multiplicity density at mid-rapidity in central Pb-Pb collisions at $\sqrt{s_{\mathrm{NN}}}=2.76$ TeV, Phys. Rev. Lett. 105 (2010) 252301

[5] ALICE Collaboration, K. Aamodt et al., Centrality dependence of the charged-particle multiplicity density at mid-rapidity in Pb-Pb collisions at $\sqrt{s_{\mathrm{NN}}}=2.76$ TeV, Phys. Rev. Lett. 106 (2011) 032301

[6] ALICE Collaboration, E. Abbas et al., Centrality dependence of the pseudorapidity density distribution for charged particles in $\mathrm{Pb}-\mathrm{Pb}$ collisions at $\sqrt{s_{\mathrm{NN}}}=2.76 \mathrm{TeV}$, Phys. Lett. B 726 (2013) 610

[7] ALICE Collaboration, B. Abelev et al., Pseudorapidity Density of Charged Particles in $p+P b$ Collisions at $\sqrt{s_{\mathrm{NN}}}=5.02$ TeV, Phys. Rev. Lett. 110 (2013) 032301

[8] ALICE Collaboration, K. Aamodt et al., Two-pion Bose-Einstein correlations in central Pb-Pb collisions at $\sqrt{s_{\mathrm{NN}}}=2.76 \mathrm{TeV}$, Phys. Lett. B $696(2011) 328$

[9] ALICE Collaboration, B. Abelev et al., Freeze-out radii extracted from three-pion cumulants in pp, p-Pb and Pb-Pb collisions at the LHC, Phys. Lett. B 739 (2014) 139 [arXiv: 1404.1194 ]

[10] ALICE Collaboration, B. Abelev et al., Multiplicity dependence of the average transverse momentum in pp, $p-P b$, and $P b-P b$ collisions at the LHC, Phys. Lett. B 727 (2013) 371

[11] ALICE Collaboration, B. Abelev et al., Production of charged pions, kaons and protons at large transverse momenta in pp and Pb-Pb collisions at $\sqrt{s_{\mathrm{NN}}}=2.76 \mathrm{TeV}$, Phys. Lett. B 736 (2014) 196 [arXiv:1401.1250] 
[12] ALICE Collaboration, B. Abelev et al., Multiplicity dependence of pion, kaon, proton and lambda production in $p-P b$ collisions at $\sqrt{s_{\mathrm{NN}}}=5.02$ TeV, Phys. Lett. B 728 (2014) 25

[13] ALICE Collaboration, B. Abelev et al., Centrality dependence of $\pi$, K, and p production in $\mathrm{Pb}-\mathrm{Pb}$ collisions at $\sqrt{s_{\mathrm{NN}}}=2.76$ TeV, Phys. Rev. C 88 (2013) 44910

[14] ALICE Collaboration, B. Abelev et al., $K_{s}^{0}$ and $\Lambda$ Production in Pb-Pb Collisions at $\sqrt{s_{\mathrm{NN}}}=2.76$ TeV, Phys. Rev. Lett. 111 (2013) 222301 [arXiv: 1307 . 5530]

[15] S. Wheaton, J. Cleymans, M. Hauer, THERMUS - A thermal model package for ROOT, Comp. Phys. Comm. 180 (2009) 84

[16] A. Andronic, P. Braun-Munzinger, J. Stachel, Thermal hadron production in relativistic nuclear collisions: The hadron mass spectrum, the horn, and the QCD phase transition, Phys. Lett. B 673 (2009) 142

[17] M. Petran, J. Letessier, J. Rafelski, G. Torrieri, SHARE with CHARM, Comp. Phys. Comm. in press [arXiv:1310.5108]

[18] ALICE Collaboration, K. Aamodt et al., Elliptic flow of charged particles in Pb-Pb collisions at 2.76 TeV, Phys. Rev. Lett. 105 (2010) 252302

[19] ALICE Collaboration, K. Aamodt et al., Higher harmonic anisotropic flow measurement of charged particles in Pb-Pb collisions at $\sqrt{s_{\mathrm{NN}}}=2.76 \mathrm{TeV}$, Phys. Rev. Lett. 107 (2011) 032301

[20] ALICE Collaboration, K. Aamodt et al., Harmonic decomposition of two-particle angular correlations in $P b-P b$ collisions at $\sqrt{s_{\mathrm{NN}}}=2.76$ TeV, Phys. Lett. B 708 (2012) 249

[21] ALICE Collaboration, B. Abelev et al., Elliptic flow of identified hadrons in Pb-Pb collisions at $\sqrt{s_{\mathrm{NN}}}=2.76 \mathrm{TeV}, \operatorname{arXiv}: 1405.4632$

[22] ALICE Collaboration, B. Abelev et al., Long-range angular correlations on the near and away side in $p-P b$ collisions at $\sqrt{s_{\mathrm{NN}}}=5.02$ TeV, Phys. Lett. B 719 (2013) 29

[23] ALICE Collaboration, K. Aamodt et al., Suppression of Charged Particle Production at Large Transverse Momentum in Central Pb-Pb Collisions at $\sqrt{s_{\mathrm{NN}}}=2.76$ TeV, Phys. Lett. B 696 (2011) 30

[24] ALICE Collaboration, B. Abelev et al., Centrality dependence of charged particle production at large transverse momentum in $\mathrm{Pb}-\mathrm{Pb}$ collisions at $\sqrt{s_{\mathrm{NN}}}=2.76$ TeV, Phys. Lett. B 720 (2013) 52

[25] ALICE Collaboration, B. Abelev et al., Transverse Momentum Distribution and Nuclear Modification Factor of Charged Particles in p + Pb Collisions at $\sqrt{s_{\mathrm{NN}}}=5.02 \mathrm{TeV}$, Phys. Rev. Lett. 110 (2013) 082302

[26] ALICE Collaboration, B. Abelev et al., Transverse momentum dependence of inclusive primary charged-particle production in p-Pb collisions at $\sqrt{s_{\mathrm{NN}}}=5.02 \mathrm{TeV}$, Eur. Phys. J. C 74 (2014) 3054 [arXiv:1405.2737]

[27] Yu. L. Dokshitzer and D.E. Kharzeev, Heavy-quark colorimetry of QCD matter, Phys. Lett. B 519 (2001) 199

[28] ALICE Collaboration, B. Abelev et al., Suppression of high transverse momentum prompt D mesons in central Pb-Pb collisions at $\sqrt{s_{\mathrm{NN}}}=2.76 \mathrm{TeV}$, JHEP 09 (2012) 112

[29] ALICE Collaboration, B. Abelev et al., Measurement of prompt D-meson production in $\mathrm{p}-\mathrm{Pb}$ collisions at $\sqrt{s_{\mathrm{NN}}}=5.02 \mathrm{TeV}$, arXiv:1405.3452

[30] CMS Collaboration, $J / \psi$ results from CMS in Pb-Pb collisions, with $150 \mu b^{-1}$ data, CMS-PAS-HIN-12-014 (2012) 
[31] ALICE Collaboration, B. Abelev et al., Azimuthal anisotropy of D-meson production in $\mathrm{Pb}-\mathrm{Pb}$ collisions at $\sqrt{s_{\mathrm{NN}}}=2.76$ TeV, Phys. Rev. C 90 (2014) 034904 [arXiv: 1405.2001 ]

[32] T. Matsui, H. Satz, J/ $\psi$ suppression by quark-gluon plasma formation, Phys. Lett. B 178 (1986) 416

[33] P. Braun-Munzinger, J. Stachel, (Non)thermal aspects of charmonium production and a new look at J/ $\psi$ suppression, Phys. Lett. B 490 (2000) 196

[34] S. Digal, P. Petreczky, and H. Satz, Quarkonium feed-down and sequential suppression, Phys. Rev. D 64 (2001) 094015

[35] ALICE Collaboration, B. Abelev et al., Centrality, rapidity and transverse momentum dependence of $J / \psi$ suppression in $P b-P b$ collisions at $\sqrt{s_{\mathrm{NN}}}=2.76$ TeV, Phys. Lett. B 734 (2014) 314

[36] PHENIX Collaboration, A. Adare et al., J/ $\psi$ suppression at forward rapidity in Au + Au collisions at $\sqrt{s_{\mathrm{NN}}}=200 \mathrm{GeV}$, Phys. Rev. C 84 (2011) 054912

[37] Upgrade of the ALICE Experiment: Letter of Intent, CERN-LHCC-2012-012; Addendum: The Muon Forward Tracker, CERN-LHCC-2013-014

[38] ALICE Upgrade Technical Design Reports: CERN-LHCC-2013-019; CERN-LHCC-2013-020; CERN-LHCC-2013-024 